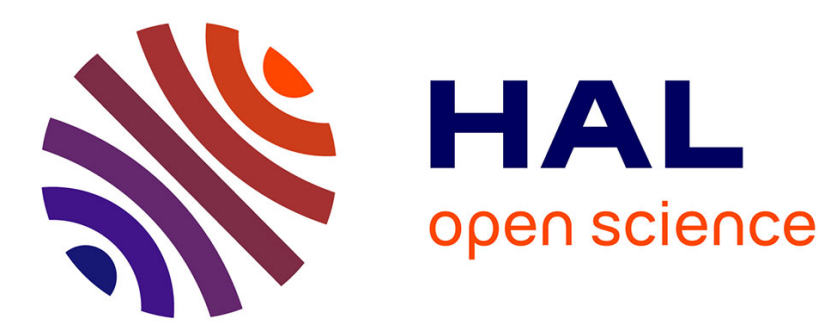

\title{
PROPRIÉTÉS DYNAMIQUES DES POLYMÈRES : FORTES ÉLONGATIONS
}

François Boué, G. Jannink

\section{To cite this version:}

François Boué, G. Jannink. PROPRIÉTÉS DYNAMIQUES DES POLYMÈRES: FORTES ÉLONGATIONS. Journal de Physique Colloques, 1978, 39 (C2), pp.C2-183-C2-191. 10.1051/jphyscol:1978231 . jpa-00217386

\section{HAL Id: jpa-00217386 https://hal.science/jpa-00217386}

Submitted on 1 Jan 1978

HAL is a multi-disciplinary open access archive for the deposit and dissemination of scientific research documents, whether they are published or not. The documents may come from teaching and research institutions in France or abroad, or from public or private research centers.
L'archive ouverte pluridisciplinaire HAL, est destinée au dépôt et à la diffusion de documents scientifiques de niveau recherche, publiés ou non, émanant des établissements d'enseignement et de recherche français ou étrangers, des laboratoires publics ou privés. 


\title{
PROPRIÉTÉS DYNAMIQUES DES POLYMĖRES : FORTES ELONGATIONS
}

\author{
F. BOUÉ et G. JANNINK \\ DPh-G/PSRM, CEN, Saclay, B.P. 2, 91190 Gif-sur-Yvette, France
}

\begin{abstract}
Résumé. - Nous présentons les résultats de diffusion des neutrons par des polystyrènes fondus, à différents stades d'une déformation uniaxiale et à différents stades du retour à l'équilibre par relaxation de contrainte.

Les dépendances de l'intensité diffusée en fonction du transfert de moment $q$ et des paramètres de l'expérience s'interprètent à l'aide de longueurs de contour caractéristiques : $n_{\mathrm{c}}$ et $n_{\mathrm{cc}}$ pour la déformation, $n_{\text {ccc }}$ pour la relaxation. Ces longueurs de contours caractéristiques correspondent à des zones d'espaces réciproques définies par $q^{*}, q_{\lambda}^{*}$ et $q^{* *}$ respectivement. La dépendance en température d'élongation de $q^{*}$ et $q_{\lambda}^{*}$ et la dépendance en temps de $q^{* *}$ sont discutées en fonction des intensités diffusées observées. Ces expériences mettent en évidence le rôle des enchevêtrements dans le comportement élastique et viscoélastique des polymères fondus.
\end{abstract}

\begin{abstract}
We present neutron scattering data from uniaxially hot stutched polystyrene samples, in different states of relaxation towards the equilibrium configuration.

Inter and intramolecular correlations are observed in separate experiments. The deformed state is determined by characteristic contour lengths along the polymer chaim, $n_{\mathrm{c}}$ and $n_{\mathrm{cc}}$. These lengths correspond to the reciprocal space distances $q^{*}$ and $q_{\lambda}^{*}$ respectively; $q^{*}$ separates the quadrupolar from the dipolar contributions to the coil deformation, and $q_{\lambda}^{*}$ is the reciprocal scale beyond which the configuration is relaxed. The scale $\left(0, q^{*}\right)$ reflects the contribution of the entanglement. According to Daoudi [1] the relaxation process at constant strain is determined by a characteristic contour length $n_{\text {cces }}$, whose time dependence reflects the interaction between the coil and the matrix. The neutron scattering data indicate that a reciprocal space distance $q^{* *}$ associated to $n_{\text {cec }}$ is observable as a function of time and temperature.
\end{abstract}

1. Introduction. - A une température $T$ supérieure à la transition vitreuse $T_{\mathrm{G}}$, les matériaux polymériques sont capables de supporter des déformations élastiques élevées.

L'énergie potentielle et la configuration de la chaîne isolée dans l'état déformé sont bien connues. Mais lorsque les chaînes forment un matériau dense, une contribution importante de l'élasticité vient de la structure même du matériau et des interactions entre chaînes. Cet effet est essentiel, mais son évaluation et son observation ont toujours été difficiles. Il en est de même pour le retour à l'équilibre de la configuration de la chaîne, après cessation de la contrainte. C'est la dépendance en temps des coordonnées de la chaîne qui reflète la structure et les interactions, mais celle-ci est difficile à observer. La possibilité d'examiner des échantillons marqués sélectivement, par diffusion des neutrons aux petits angles, modifie considérablement les termes dans lesquels le problème de la structure et la relaxation se pose à l'expérimentateur. Nous exposerons les expériences faites par le groupe CRM Saclay, sur le polystyrène fondu, uniaxialement déformé, et nous essayerons d'interpréter les résultats obtenus dans les paragraphes suivants. Auparavant, nous rappelons brièvement quelques manifestations essentielles $\mathrm{du}$ comportement macroscopique des matériaux poly- mériques soumis à une déformation. Ces lois de comportement sont très différentes :

1) suivant que la déformation est forte ou faible;

2) suivant que le matériau est réticulé ou non réticulé.

a) Soit un matériau polymérique soumis à déformation uniaxiale élastique $\lambda=L / L_{0}$, où $L$ et $L_{0}$ sont les longueurs après et avant déformation; la relaxation entre la contrainte $\sigma$ et l'allongement $\lambda$ n'est pas linéaire aux grandes extensions, elle est approximativement [2]

$$
\sigma=E / 3\left(\lambda^{2}-1 / \lambda\right) \quad \text { (Neo Hooke) }
$$

où $E$ est le module d'Young. La déviation de $\sigma$ par rapport à $(1.1)$ est plus forte pour les matériaux réticulés que pour les matériaux non réticulés. Aux faibles déformations, $\sigma$ est proportionnel à $\varepsilon=\lambda-1$

$$
\sigma=E / 3 \varepsilon, \quad . \varepsilon \rightarrow 0 \quad \text { (Hooke) }
$$

(dans le cas d'un cisaillement, la réponse reste toujours linéaire).

b) Soit un matériau non réticulé, fondu, soumis à un gradient de vitesse longitudinale; d'une manière semblable à la situation (1.2), on a pour les faibles sollicitations

$$
\sigma=3 \eta s \quad \text { où } \quad s=(1 / \varepsilon) \mathrm{d} \varepsilon / \mathrm{d} t
$$


et où $\eta$ est la viscosité dans un écoulement à gradient transversal. La relation (1.3) est la loi de Trouton, qui décrit le comportement rhéologique à la limite des gradients nuls. Lorsque le gradient de vitesse augmente, le rapport $\sigma / s$ augmente considérablement par rapport à la valeur limite $3 \eta$.

Le changement est encore plus net dans le cas de la chaîne polymérique en solution très diluée : aux faibles gradients $s$, la déformation de la chaîne n'est pas affine aux lignes d'écoulement. Au-delà d'une valeur critique $s=s_{\mathrm{c}}=1 / \tau_{1}$ (où $\tau_{1}$ est le temps de relaxation du premier mode, typiquement de l'ordre de $10^{-4} \mathrm{~s}$ pour une masse moléculaire $M=10^{5} \mathrm{dal}-$ tons), la configuration de la chaîne passe subitement de l'état pelote déformée à l'état étiré. L'alignement par écoulement avec gradient de vitesse longitudinale se produit non seulement en solution, mais aussi dans les liquides polymériques. Le gradient de vitesse nécessaire $\tilde{s}$ est beaucoup plus faible que $s_{\mathrm{c}}$. Selon Peterlin $\tilde{s}=s_{\mathrm{c}}[\eta] / \eta$, où $[\eta]$ est la viscosité spécifique du polymère en solution et $\eta$ la viscosité du polymère liquide. (Il faut noter cependant que le temps nécessaire à l'alignement augmente dans des proportions semblables). Cette diminution considérable fait apparaître un effet coopératif entre chaîne, dû aux interactions de simple contact répulsif et d'enchevêtrement. Par ailleurs, ces enchevêtrements sont responsables de la réponse élastique des matériaux polymériques non réticulés. Suivant l'échelle des temps considérés, les matériaux polymériques non réticulés soumis à une contrainte ont donc un comportement de solide élastique ou de liquide. Ils ont, en plus, un comportement qui les distingue des autres matériaux et que l'on appelle viscoélastique. Entre les temps très courts $\left(t \leqslant t_{1} \sim 10^{-6} \mathrm{~s}\right)$ de réponse à une sollicitation donnée, et les temps intermédiaires caractéristiques du plateau caoutchoutique $\left(t \geqslant t_{2} \sim 10^{-2} \mathrm{~s}\right)$, le module élastique décroît d'un facteur de l'ordre de $10^{3}$. De part et d'autre de $\left(t_{1}, t_{2}\right)$ le comportement est élastique ; dans l'intervalle $\left(t_{1}, t_{2}\right)$, il est viscoélastique. Le comportement viscoélastique est surtout connu à la limite des déformations ou des sollicitations infinitésimales. Lorsque les amplitudes sont finies, le comportement viscoélastique est profondément modifié par rapport au cas infinitésimal.

En particulier la réponse n'est plus linéaire en $s$ (ou en $\mathrm{d} \sigma / \mathrm{d} t$ ). Ce comportement viscoélastique aux fortes déformations est moins bien connu. Cependant, un grand nombre d'observations ont été faites sur des échantillons déformés à gradient de vitesse constant (de Vries [5]). Ce dispositif permet l'étude du comportement en fonction de trois paramètres significatifs

$$
\lambda, s, T \text {. }
$$

La difficulté inhérente à l'observation des fortes déformations reste néanmoins le contrôle des temps $\widetilde{T}$ nécessaire à l'établissement des configurations d'équilibre, pour une contrainte $s$ donnée. Or les échantillons que nous avons examinés ont été étirés uniaxialement pendant un temps suffisamment long pour que l'allongement soit fini $(\lambda \sim 3)$, mais suffisamment petit pour que sa recouvrance soit totale après cessation de la contrainte. La déformation est élastique (ce qui n'exclut pas un comportement viscoélastique). Les chaînes ne sont pas alignées au sens décrit précédemment, elles sont néanmoins fortement déformées, de sorte que la déformation est observable. Jusqu'à maintenant, les observations avaient été faites à deux échelles de distance.

a) A l'échelle macroscopique, par mesure de $L$ en fonction du temps (ou de tout autre distance repérable par 2 traits gravés, donc de l'ordre du micron).

b) A l'échelle microscopique, par mesure de l'orientation du segment statistique de la chaîne polymérique (de l'ordre de $10 \AA$ ). Entre ces deux échelles, les corrélations de distance allant de 10 à $10^{3} \AA$ n'ont été mesurées que tout récemment. La sonde neutronique permet l'exploration de cet intervalle. Nous examinons les diagrammes de diffusion centrale par des échantillons qui sont des plaquettes de polystyrène de masse moléculaire $M=10^{5}$ daltons. La longueur initiale est $L_{0} \simeq 3 \mathrm{~cm}$. Le gradient de vitesse est $s=0,18 \mathrm{~s}^{-1}$. Les différentes températures d'étirage sont $T=115^{\circ}, 120^{\circ}, 130^{\circ}$ et $140^{\circ} \mathrm{C}$.

Un des problèmes posés par la déformation. des matériaux polymériques dans le domaine $(\lambda, T, s)$ défini ci-dessus, est le rôle des enchevêtrements dans leur comportement.

1) Déformation élastique. Les enchevêtrements sont responsables du comportement élastique des matériaux non réticulés. Chacun d'eux est un centre de forces agissant sur les chaînes polymériques. La relation (1.1) attribue à ces forces une distribution uniforme dans le volume de l'échantillon. Cependant, ces enchevêtrements seraient plus localisés selon certaines interprétations de mesures dynamiques. L'observation des corrélations de distance peut-elle contribuer à la solution de ce problème ?

2) Comportement viscoélastique. Une équivalence entre l'effet de température $\left(T-T_{\mathrm{G}}\right)$ et des gradients de vitesse a été observée à l'échelle de la déformation macroscopique. En particulier le module élastique croît avec $s$ et avec $\left(T-T_{\mathrm{G}}\right)^{-1}$. Cette équivalence est-elle uniformément valable à toutes les échelles de distances comprises entre $10^{3}$ et $10 \AA$ ?

\section{3) Retour à l'équilibre : relaxation}

a) Par cessation de la contrainte; de la dépendance en temps de la recouvrance $L(t) \rightarrow L_{0}$, on détermine le spectre de retardation, faisant apparaître en particulier un temps caractéristique long $\left(t \simeq 10^{2} \mathrm{~s}\right)$. Ce temps a-t-il la même signification à toutes les échelles de distances?

b) Par relaxation de la contrainte à déformation constante. Daoudi et de Gennes [1] ont calculé (pour 
une chaine libre dans un gel déformé) des temps de relaxation associés au retour à l'équilibre, qui varient avec la distance de corrélation. A un instant donné, quelles sont les recouvrances aux différentes échelles de distance d'une chaîne du matériau non réticulé ?

\section{Remarques sur la diffusion des neutrons. -} Nous reprenons une notation de Duplessix [6] dans son étude des gels réticulés.

a) Les échantillons A sont des mélanges de $99 \%$ de chaînes protonées et $1 \%$ chaînes deutérées. La diffusion des neutrons $S_{1}(q)$ (normalisée à l'unité) par de tels échantillons permet de mesurer :

1) Le rayon de la chaîne polymérique $R_{\mathrm{G}}(\Omega)$ dans les différentes directions $\Omega$ entre l'étirage et le transfert de moment $q$ (la limite d'appareillage $q_{\min } \simeq 6 \times 10^{-3} \AA^{-1}$ ne nous permet pratiquement que la mesure des rayons transverses à la direction d'étirement).

2) La fonction de corrélation des paires de segments de la chaîne polymérique, dans l'intervalle $R_{\mathrm{G}}^{-1} \ll q_{\Omega}<l^{-1}$, dans chacune des directions $\Omega$.

b) Les échantillons de type B (proposés récemment par J. P. Cotton pour l'étude de la déformation des échantillons non réticulés) sont constitués de $100 \%$ de chaînes partiellement deutériées; la chaîne est composée de 3 séquences, dont la centrale est marquée.

Le signal $S(q)$ diffusé peut alors se concevoir :

1) Comme une fonction [7] de corrélation des distances entre séquences de chaînes marquées, si celles-ci forment un réseau dans le volume de l'échantillon. On a

$$
S(q)=\frac{1}{1-S_{0}(q)}
$$

où $S_{0}(q)$ est la transformée de Fourier de la fonction de corrélation de paire de deux nauds voisins $g(r)$

$$
g(r)=\mathrm{e}^{-r^{2} /\left\langle\tilde{r}^{2}\right\rangle} \mathrm{e}^{-V(r) / k T} \quad \text { (de Gennes) }
$$

où $V(r)$ est le potentiel d'interaction effective.

La fonction $S(q)$ (2.1) indique un maximum à $q=\tilde{q}$. L'intensité, la largeur du pic et la valeur de $\tilde{q}$ sont tous trois fonction de l'interaction répulsive effective $V(r)$ entre séquences de chaînes marquées.

2) Comme une mesure du rayon des séquences de chaînes marquées. Si les chaînons sont distribués d'une manière uniformément aléatoire, la position du maximum de l'intensité diffusée est [8]

$$
\tilde{q}=1 / \sqrt{R_{\mathrm{d}}^{2}}
$$

où $R_{\mathrm{d}}^{2}$ est le rayon de giration de la séquence marquée.

Dans l'état non étiré [9], les séquences marquées sont effectivement distribuées d'une manière homogène (absence de corrélations). La seconde inter- prétation est donc correcte. Dans l'état étiré, la présence éventuelle de points d'enchevêtrements localisant les forces de déformation de la chaîne ne permet pas de décider a priori quelle est la bonne interprétation.

3. Comportement élastique. - a) I. Ward [2] remarque que la relation (1.1) s'obtient sans faire appel à la notion de polymère. Il suffit en effet :

1) De supposer que la déformation est homogène à toute échelle. Soit par exemple une transformation $u(x)$, qui à chaque point $x$ fait correspondre $x+u$. Les longueurs $\mathrm{d} x^{2}$ des éléments infinitésimaux $\mathrm{d} x$ deviennent

$$
\begin{aligned}
\overline{\mathrm{d}} x^{2}=\mathrm{d} x^{2}\left(1+2\left(\frac{\partial u}{\partial x}+\frac{1}{2}\right.\right. & \left.\left.\frac{\partial^{2} u}{\partial x^{2}}\right)\right)= \\
& =\mathrm{d} x^{2}\left(1+2 e_{x x}\right)
\end{aligned}
$$

L'hypothèse d'homogénéité exprime que chaque longueur finie $l_{x}$ est transformée en

$$
\bar{l}_{x}^{2}=l_{x}^{2}\left(1+2 e_{x x}\right)=\lambda_{x}^{2} l_{x}^{2}
$$

La relation (3.2) est aussi appelée hypothèse de déformation affine.

2) De se donner une équation constitutive la plus générale possible (relation entre la contrainte et la déformation)

$$
1+2 e_{x x}=\frac{B}{E}\left(\sigma_{x x}-p\right) \quad \text { (Rivlin) }
$$

où $p$ est une pression arbitrairement fixée à $p=E / 3$ pour une contrainte nulle.

3) De supposer l'incompressibilité

$$
e_{x x}+e_{y y}+e_{z z}=0 .
$$

Ces conditions suffisent à établir la relation (1.1).

$$
\sigma_{x x}=E / 3\left(\lambda_{x}^{2}-1 / \lambda_{x}\right)
$$

dans le cas de l'étirage uniaxial dans la direction $x$.

b) Il n'est pas nécessaire de supposer une homogénéité allant de l'échelle infinitésimale à l'échelle macroscopique (c'est-à-dire $L_{0}$ ) pour obtenir une relation du type (1.1). Dans le cas des caoutchoucs réticulés, Kuhn et Grün [10] supposent une affinité depuis l'échelle de distance entre points de réticulation : l'entropie d'une chaîne déformée peut s'écrire

$$
\bar{S}=C-k\left(\left\langle r_{0}^{2}\right\rangle\right)^{-1}\left(\bar{x}^{2}+\bar{y}^{2}+\bar{z}^{2}\right)
$$

où $\bar{x}, \bar{y}, \bar{z}$ sont les coordonnées du segment entre deux points de réticulation voisins et où $C$ est une constante. La loi d'homogénéité implique

$$
\bar{x}^{2}=\lambda_{x}^{2} x_{0}^{2}, \text { etc. . }
$$


La différence d'entropie entre les chaînes déformées et les chaînes au repos est alors

$$
\Delta S=-\frac{1}{2} v k\left(\lambda_{x}^{2}+\lambda_{y}^{2}+\lambda_{z}^{2}-3\right)
$$

où $v$ est la densité de points de réticulation.

La force associée à $\Delta S$ est

$$
\begin{aligned}
& f=-T \partial(\Delta S) / \partial L=-T / L_{0} \partial(\Delta S) / \partial \lambda_{x} \\
& \sigma=f \lambda_{x}^{-1}=v k T\left(\lambda_{x}^{2}-1 / \lambda_{x}\right)=E\left(\lambda_{x}^{2}-1 / \lambda_{x}\right) .
\end{aligned}
$$

La relation (3.8) est analogue à (3.1); le module $\tilde{E}$ est cependant différent du module d'Young. Il est proportionnel ici à la densité d'enchevêtrement mais il est en fait inversement proportionnel à la longueur de coupure d'homogénéité, qui dans le cas des caoutchoucs, est supposée égale à $(1 / v)$.

c) On a pu observer, depuis longtemps, l'existence d'une telle coupure d'homogénéité. Par exemple, la biréfringence mesurée pour les caoutchoucs étirés est anormalement faible : la déformation à l'échelle du segment statistique $(10 \AA)$ n'est pas affine, alors qu'à l'échelle du micron elle est effectivement affine. La biréfringence met en évidence une coupure, mais ne peut mesurer la longueur caractéristique qui lui est associée. Quelle est donc cette longueur ? La technique de diffusion des neutrons permet l'exploration des distances entre $10 \AA$ et $10^{3} \AA$. Nous avons calculé l'intensité diffusée $S_{1}(q)$ d'un échantillon du type A (c'est-à-dire comportant quelques chaînes marquées) dans la direction $q$ parallèle à l'élongation

$$
S_{1}(q)=\sum_{n=1}^{N-1}(N-n) \exp \left(-q^{2}\left\langle r_{n}^{2}\right\rangle\right)
$$

où $n$ est le nombre de segments entre deux segments $i, j$ de la chaîne et où $N$ est le nombre total de segments. Soit une distance $n$ séparant deux segments $i$ et $j$ le long d'une chaîne, et une structure d'enchevêtrement définie par sa densité et sa durée de vie. On peut définir [4] une

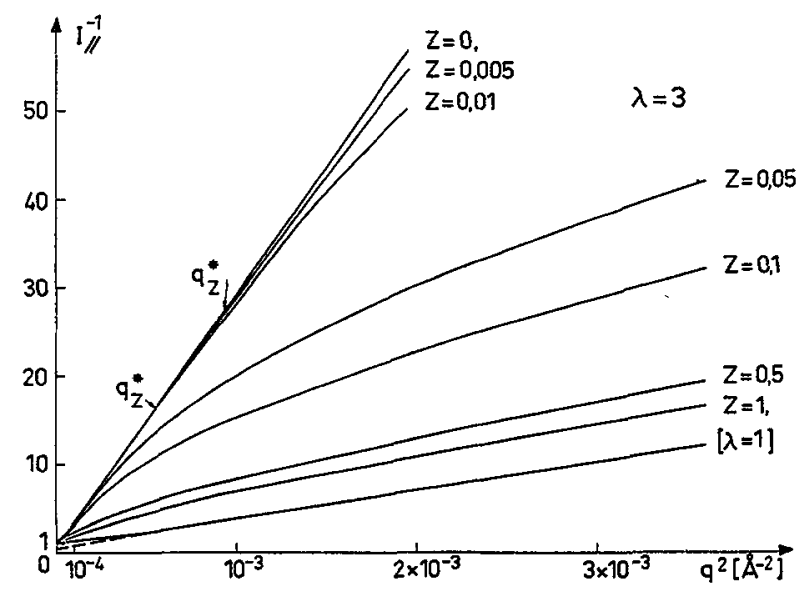

FIG. 1. - Lois de diffusion dans la direction longitudinale, en fonction du paramètre $Z=n_{\mathrm{c}} / N$, pour une chaîne de rayon $R_{\mathrm{G}} \simeq 80 \AA$ avec un allongement $\lambda=3$ (équation (3.11)). L'abscisse est le carré du transfert de moment $q=4 \pi / \lambda \sin \theta / 2$, où $\lambda$ est la longueur d'onde du neutron incident, $\theta$ l'angle de diffusion. L'ordonnée est l'inverse de l'intensité diffusée selon le calcul (3.11). distance de contour efficace, associée à une structure d'enchevêtrement, comme la plus petite distance $n=n_{\mathrm{c}}$ telle que cette structure impose une déformation affine à $r_{i j}$. Nous avons introduit une longueur de contour $n_{\mathrm{c}}$, telle que la déformation soit homogène au sens (3.2) pour tout $\left\langle r_{n}^{2}\right\rangle$ tel que $n \geqslant n_{\mathrm{c}}$. Pour $n$ inférieure à $n_{\mathrm{c}}$, nous écrivons selon Katchalsky et al.

$$
\left\langle r_{n}^{2}\right\rangle=\left(\left(\lambda^{2}-1\right) \frac{n^{2}}{n_{\mathrm{c}}^{2}}+\frac{n}{n_{\mathrm{c}}}\right) l^{2} n_{\mathrm{c}}
$$

ce qui traduit l'allongement d'une séquence de $n_{\mathrm{c}}$ chấnons fantômes, soumis à une force $f$ à ces extrémités. Notons la valeur caractéristique $n_{\mathrm{cc}}=n_{\mathrm{c}}\left(\lambda^{2}-1\right)^{1 / 2}$ qui sépare les dépendances quadratique et linéaire de $\left\langle r_{n}^{2}\right\rangle$ en fonction de $n$. On a dans la direction longitudinale $q^{\|}$

$$
\begin{aligned}
S_{1}\left(q^{\|}\right)=\sum_{n=1}^{n_{\mathrm{c}}-1}(N-n) \times \\
\quad \times \exp \left[-l^{2} n_{\mathrm{c}}\left(\left(\lambda_{x}^{2}-1\right) \frac{n^{2}}{n_{\mathrm{c}}^{2}}+\frac{n}{n_{\mathrm{c}}}\right)\right]+ \\
\quad+\sum_{n=n_{\mathrm{c}}}^{N-1}(N-n) \exp -l^{2} \frac{\lambda_{x}^{2} n}{N},
\end{aligned}
$$

Dans la direction $q^{\perp}$, perpendiculaire à l'élongation

$$
S_{1}\left(q^{\perp}\right)=\sum_{n-1}^{N-1}(N-n) \exp \left[-\frac{l^{2} n}{\lambda}\right]
$$

La figure 1 montre les courbes $S_{1}^{-1}(q)$, pour $\lambda=3$, à différentes valeurs de $n_{\mathrm{c}} / N=Z$, en fonction de $q^{2}$, pour $q R_{\mathrm{G}}>1$. Pour un matériau déformé d'une manière uniformément homogène au sens (3.2) $\left(n_{\mathrm{c}}=1, Z=0\right) S_{0}^{-1}(q)$ est proportionnel à $q^{2}$ dans toutes les directions $\Omega$ entre le vecteur $q$ et l'élongation. La dépendance en $q^{2}$ est une caractéristique de la marche au hasard du cheminement au long de la chaîne. La pente $S_{1}^{-1}(q) / q^{2}$ est proportionnelle au carré du pas de la marche au hasard. Ce pas est $\lambda_{x}^{2} l^{2}$ dans le sens longitudinal et $\frac{1}{\lambda_{x}} l^{2}$, dans le sens transversal. Notons que lorsque $\lambda_{x}=1$, les deux fonctions $S_{1}^{-1}\left(q^{\|}\right)$et $S_{1}^{-1}\left(q^{\perp}\right)$ se confondent en une seule.

En introduisant une coupure $Z=n_{\mathrm{c}} / N$ sur le contour de la chaîne, nous introduisons un changement graduel dans la loi d'homogénéité des déformations de distance, auquel on peut associer une valeur $q^{*}=n_{\mathrm{c}}^{-1 / 2} l^{-1}$. La seconde coupure

$$
n_{\mathrm{cc}}=n_{\mathrm{c}}\left(\lambda^{2}-1\right)^{-1}
$$

introduit un second changement de comportement à $q_{\lambda}>q^{*}$, entre celui d'une chaîne allongée et celui d'une marche au hasard isotrope. Ce changement s'observe plus facilement à $n_{\mathrm{c}}=N(Z=1)$; on a alors $S_{1}^{-1} \propto q$, pour $q<q_{\lambda}^{*}$ et $S_{1}^{-1} \propto q^{2}+x^{2}$ pour $q>q_{\lambda}^{*}$. Au-delà de $q_{\lambda}^{*}$ les chaînons des séquences $n_{\mathrm{cc}}$ adoptent une configuration non orientée au sens des $\left\langle\cos ^{2} \omega\right\rangle$. Cette relaxation à courte échelle se 

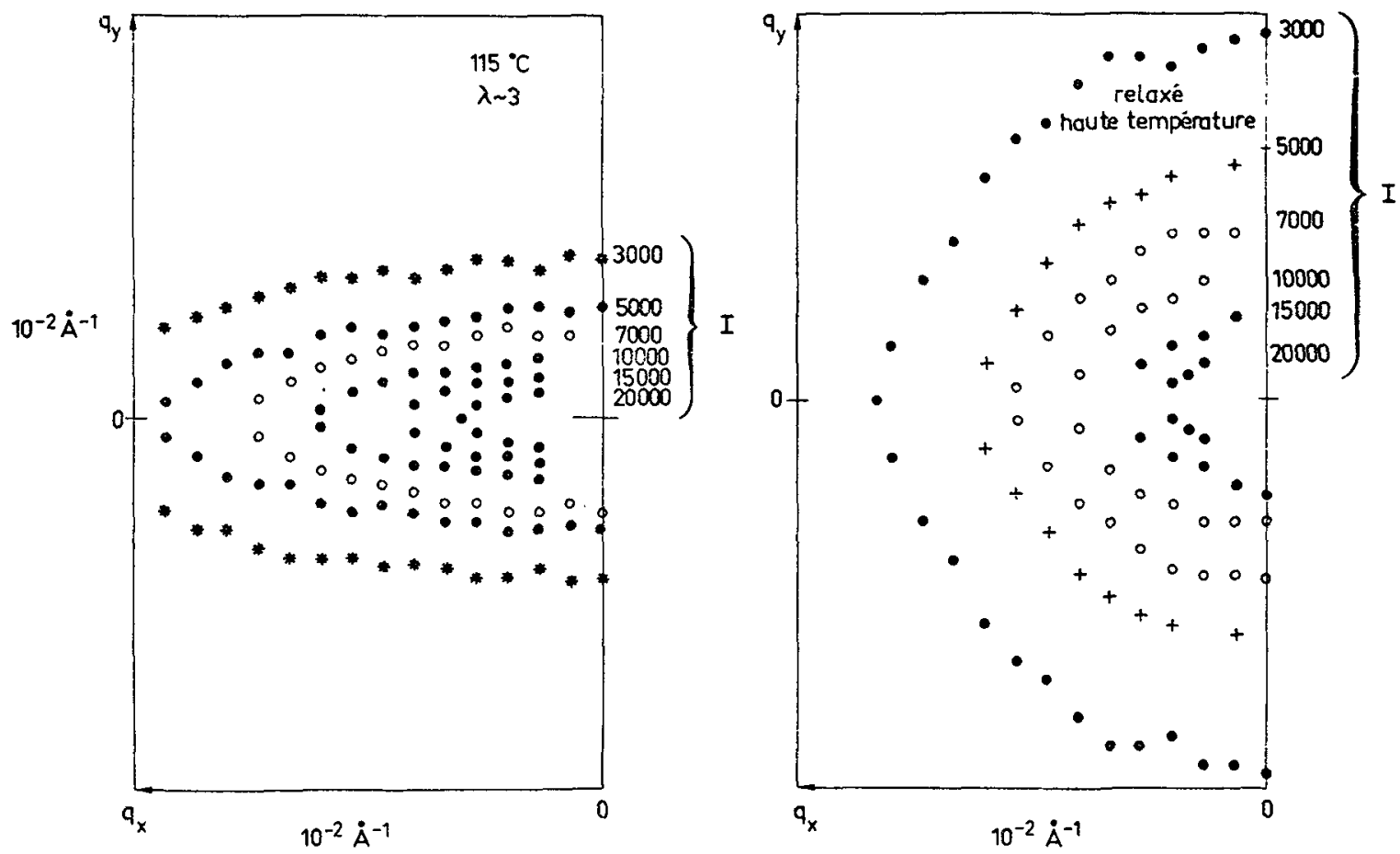

Frg. 2. - Lignes d'iso-intensité mesurées sur le détecteur bidimensionnel du canal $\mathrm{D}_{17}$ à l'Ill Grenoble. Les coordonnées $q_{x}$ et $q_{y}$ sont les projections du vecteur $q$, respectivement sur l'axe parallèle à l'allongement et perpendiculaire. La figure de gauche correspond à un échantillon immédiatement trempé après allongement uniaxial, $\lambda=3$. La figure de droite, à une trempe différée après allongement $\lambda=3$, la contrainte étant presque relaxée.

manifeste par un comportement parallèle des droites $S_{1}\left(q^{\|}\right)($pour $\lambda=3)$ et $S_{1}(q)$ pour $\lambda=1$, à partir de $q=q_{\lambda}^{*}$.

d) Les résultats expérimentaux sont enregistrés sur un détecteur bidimensionnel dont la surface est une portion de l'espace réciproque $\left(q_{x}, q_{y}\right)$. Les lignes d'isointensité de l'intensité diffusée sont reportées sur la figure 2. En prélevant les intensités le long des directions $q^{\|}=q_{y}$ et $q^{\perp}=q_{x}$, nous obtenons le diagramme de la figure 3 qui correspond à un étirage effectué à $T=115^{\circ} \mathrm{C}$. Nous remarquons tout d'abord l'homogénéité de la déformation jusqu'à

$$
q^{*}=3 \times 10^{-2} \AA^{-1},
$$

puis le fléchissement caractéristique de $S_{1}(q)$ pour des valeurs $q>q^{*}$. Notons que dans le sens transversal, $S_{1}\left(q^{\perp}\right)$ est indiscernable d'une loi de déformation homogène. Nous voyons sur cette figure qu'un matériau élastique non réticulé se déforme d'une manière homogène (affine) jusqu'à un certain point, comme si nous avions introduit des forces localisées fictives. Ce comportement est un effet des enchevêtrements.

$e)$ Dépendance de $q^{*}$ avec l'allongement $\lambda$. L'existence de $n_{\mathrm{c}}$ pour un matériau non réticulé, due à la seule présence des enchevêtrements, incite à proposer un $n_{\text {c }}$ différent de $N$ pour les matériaux (où $N$ est le nombre de segments entre deux réticulations successives). Soit donc un caoutchouc réticulé, de type A

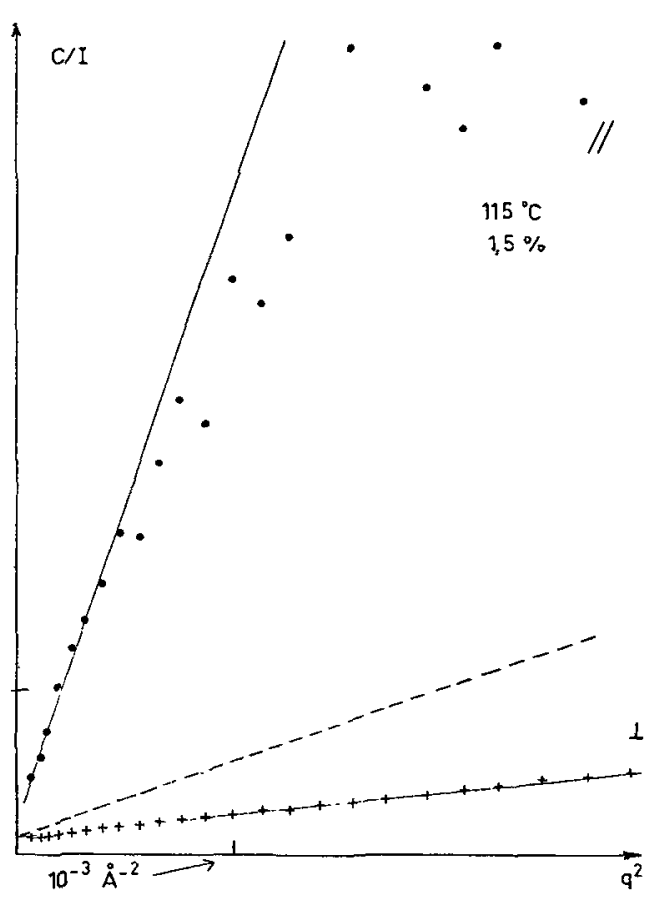

FIG. 3. - Intensités diffusées, dans les directions longitudinale (ii) et transversale $(L)$ pour un échantillon étiré à $T=115^{\circ} \mathrm{C}$ avec un allongement $\lambda=3$. Les traits continus indiquent les lois de diffusion rapportées à une déformation affine. Le trait discontinu est la loi de diffusion de l'échantillon non déformé. 


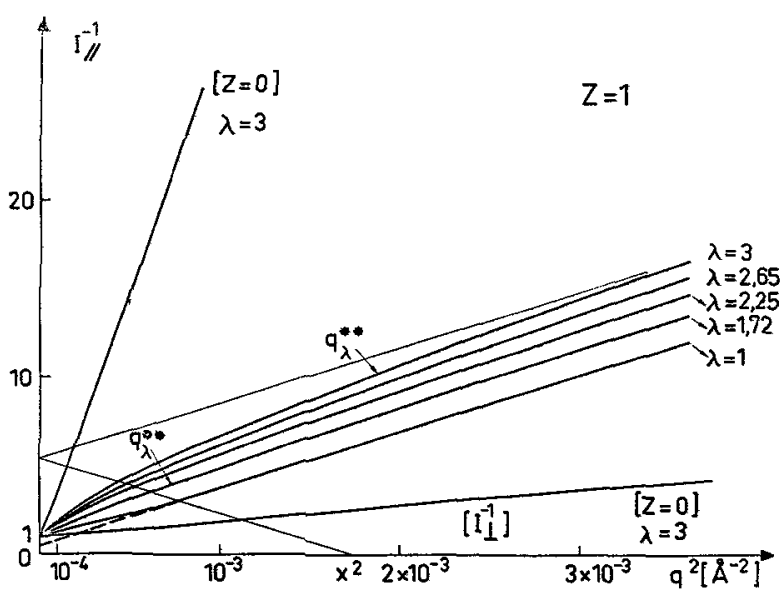

FIG. 4. - Lois d'intensité diffusées selon la formule (3.11) pour $Z=n_{\mathrm{c}} / N=1$ à différents allongements $\lambda$; on remarque la relaxation de l'orientation lorsque $q \geqslant q_{\lambda}^{*}$.

c'est-à-dire où quelques chaines entre points réticulés sont deutériées. Selon les théories classiques nous devrions avoir le schéma de la figure 4 correspondant toujours à $n_{\mathrm{c}}=N\left(Z=n_{\mathrm{c}} / N=1\right)$. Nous pensons au contraire que la présencé des enchevêtrements imposera une loi $S_{1}$ type de la figure 1 pour $Z=n_{\mathrm{c}} / N<1$. Dans ce cas, la relation contrainte-allongement devient

$\sigma=n_{\mathrm{c}}^{-1} k T\left(\lambda_{x}^{2}-1 / \lambda_{x}\right) \neq N^{-1} k T\left(\lambda_{x}^{2}-1 / \lambda_{x}\right)$.

Il est clair que la dépendance de $q^{*}$ avec $\lambda$ sera différente de $1 / \lambda$.

$\sigma=n_{c}^{-1}(\lambda) k T\left(\lambda_{x}^{2}-1 / \lambda_{x}\right)=E(\lambda)\left(\lambda_{x}-1 / \lambda_{x}\right)$.

La variation $E(\lambda)$ est connue expérimentalement. Des mesures systématiques de $n_{\mathrm{c}}(\lambda)$ permettraient d'établir une relation du type (3.14). Pour un matériau non réticulé, il a été vérifié que $E$ ne dépend pas de $\lambda$. On peut donc poser en première approximation $n_{\mathrm{c}}$ indépendant de $\lambda$, ou encore $q^{*} \propto \lambda^{-1}$.

f) Echantillons du type B. Nous retenons du diagramme expérimental de la figure 3 que la déformation est affine au moins jusqu'à l'échelle des rayons de giration $\left(q \sim R_{\mathrm{G}}^{-1}\right.$ ). Une confirmation de cette observation est donnée par le diagramme de diffusion par l'échantillon de type B (Fig. 5). Le lieu des maxima des intensités diffusées décrit une courbe dont la forme est approximativement une ellipse, dont l'ellipticité est rigoureusement conforme à l'affinité $e=\lambda_{x}^{3 / 2}-1=2,9$ jusqu'à l'échelle de distance $q^{-1}=160 \mathrm{~A}$. Lincerprétatión de cette observation n'est pas immédiate. Pèarson [11] a montré récemment que les points de rétiçulation sont, plus localisés dans l'état étiré que dans l'état relaxé. 'Dans la mesure où $n_{\mathrm{c}}$ correspond

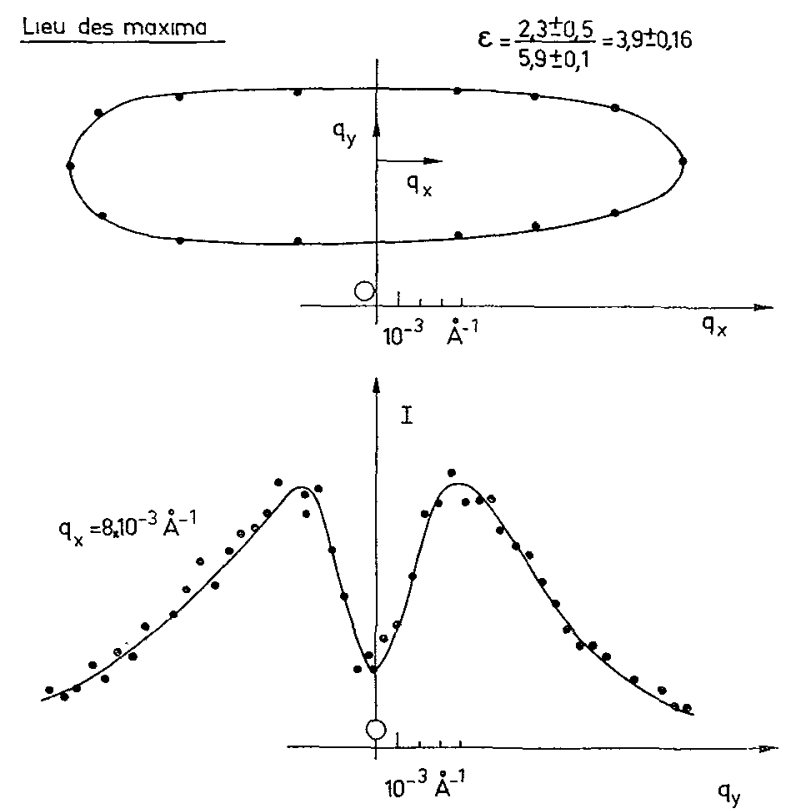

FIG. 5. - Résultat de l'expérience B : Vue du lieu des maxima sur le détecteur bidimensionnel. Dépendance de l'intensité diffusée en fonction du transfert de moment $q_{y}$.

à des points de réticulation fictifs, lexpérience $B$ s'interprète alors dans le sens d'une distance entre séquences marquées.

4. Comportement viscoélastique. - Pour une déformation périodique infinitésimale, le module élastique prend deux valeurs $E_{1}^{\prime}$ et $E_{2}^{\prime}$ suivant le domaine de fréquence considéré. Dans une expérience d'étirage définie par les trois paramètres $\sigma, \lambda(t)$ et $T$, on retrouve le phénomène viscoélastique-sous la forme analogue de la succession de deux modules élastiques : $E_{1}^{\prime}$ aux temps courts $\left(10^{-3}\right.$ s) et $E_{2}^{\prime}$ aux temps longs $\left(10^{-2} \mathrm{~s}\right)$, le rapport des deux modules étant

$$
E_{1}^{\prime} / E_{2}^{\prime} \simeq 10^{3} \text {. }
$$

Dans le cas des déformations de fortes amplitudes, définies par les paramètres $s, \lambda(t)$ et $T$, nous n'avons pas trouvé d'observations qui mettent en évidence cet aspect de la viscoélasticité. Deux autres manifestations de la viscoélasticité sont cependant connues, dans le cas de l'expérience qui nous concerne ici. Il s'agit :

a). De la décroissance du module $E$ en fonction de la température $T-T_{\mathrm{G}}$, à gradient fixé $s$.

b) De la superposition des effets de variation de température $T-T_{\mathrm{G}}$ à gradient fixé, et de ceux de la variation de gradients à $T-T_{\mathrm{G}}$ fixé, sur la biréfringence. On a [12]

$$
\Delta n=f_{n}\left(a_{T}^{n} s\right)
$$


où $a_{T}$ est une fonction décroissante de la température. De même

$$
E=f_{E}\left(a_{T}^{E} s\right) .
$$

Dans la théorie [4] WLF, la fonction $a_{T}$ est universelle, elle diverge à la température $T \simeq T_{\mathrm{G}}-50^{\circ}$.

Nous avons déterminé les rayons de giration transferses et les corrélations de paires $S_{1}(q)$ aux différentes températures d'étirage $\widetilde{T}=115^{\circ}, 120^{\circ}, 130^{\circ}$ et $140^{\circ}$ à $s=0,18 \mathrm{~s}^{-1}$ et $\lambda=3$. Sur la figure 6 , les rayons de giration sont donnés par la pente de l'inverse de l'intensité diffusée $S_{1}^{-1}$ en fonction de $q^{2}$. Le diagramme montre que le rayon ne dépend pas de la température. Sa valeur correspond à celle de la déformation affine. Les fonctions de corrélation $S_{1}^{-1}(q)$ dans le domaine intermédiaire

$$
10^{-2} \AA^{-1} \leqslant q \leqslant 10^{-1} \AA^{-1}
$$

indiquent par contre une dépendance marquée en température (Fig. 7).

1) $q^{*}$ diminue lorsque $T-T_{\mathrm{G}}$ augmente. On a $n_{\mathrm{c}} \propto q^{*-1}$ et on peut supposer

$$
n_{\mathrm{c}} \propto E^{-1}=f_{E}^{-1}\left(a_{T}^{E} s\right) .
$$

2) Dans l'intervalle $\left(0, R^{-1}\right)$ la dépendance en $T, s$ de l'intensité peut s'écrire

$$
S_{1}(q)=S_{1}\left(q, a_{T}^{s} s\right) .
$$

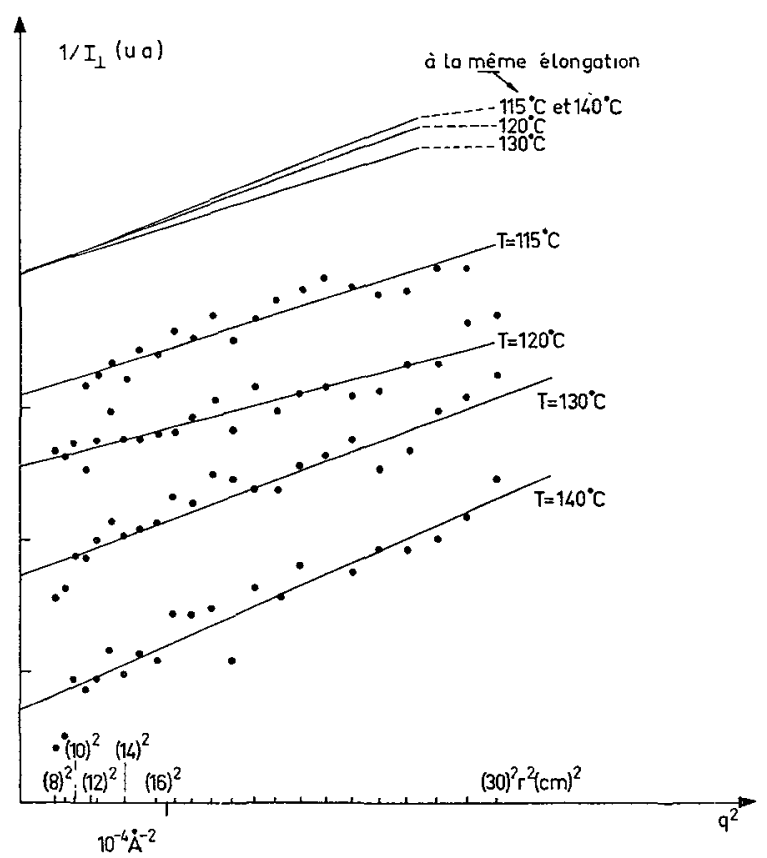

FIG. 6. - Intensıtés diffusées dans le domaine de Guınıer, dans la direction transversale, pour différentes températures d'étirage. Le diagramme du haut est une normalisation des intensités diffusées au même allongement $\lambda=3$. Les pentes de $S^{-1}$ sont les rayons de giration transversals.

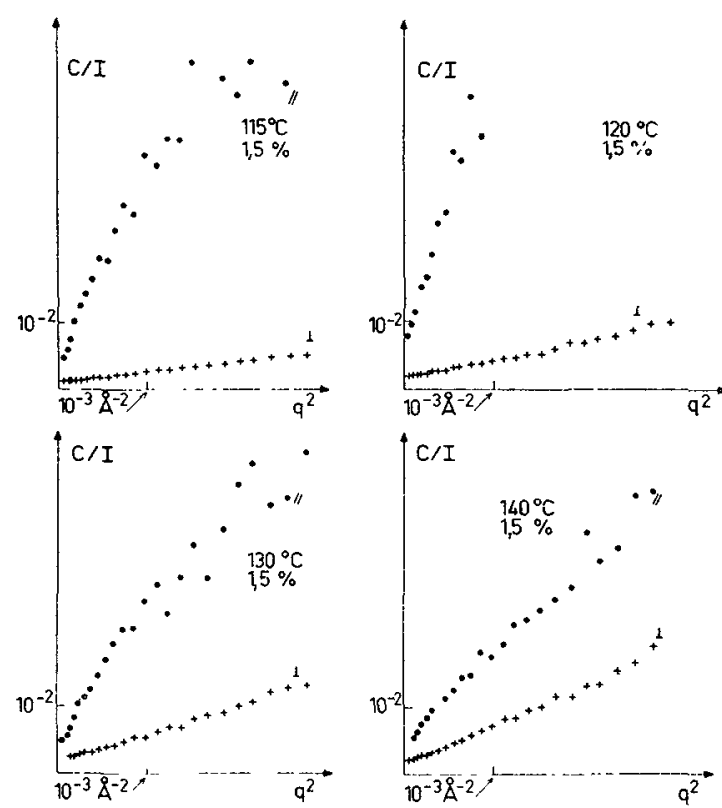

Fig. 7. - Intensités diffusées dans le domaine intermédiaire, dans les directions transversales et longitudinales, pour différentes températures d'étirage $(\lambda=3)$.

Dans l'intervalle $q \geqslant R^{-1}$, la relation est moins simple, car la figure 7 montre que $a_{r}$ est nécessairement une fonction décroissante de $q$. Le résultat essentiel de la figure 7 est l'accroissement de $n_{\mathrm{c}}$ avec la température. Reprenons la définition de $n_{\mathrm{c}}$, comme la plus petite longueur de contour pour que deux segments $i, j$ (tels que $i-j=n_{\mathrm{c}}$ ) soient déformés d'une manière affine, pour une structure donnée d'enchevêtrement entre $i$ et $j$. Lorsque la température $T$ augmente, le temps de vie de cette structure devient trop faible. Il faut incorporer un plus grand nombre de segments pour obtenir une structure d'enchevêtrements de durée de vie suffisante. Cette durée de vie $\tau_{i j}$ est une fonction décroissante de la température. On associe la diminution du module $E$ entre les valeurs élevées de l'argument $a_{T} s$ et les valeurs faibles, à une transitionvitreuse. Si on applique cette notion à la variation de $n_{\mathrm{c}}$, il existerait pour $s=0,18 \mathrm{~s}^{-1}$ un domaine de température $T$ au-delà des températures indiquées figure 7 , dans lequel $n_{\mathrm{c}}$ serait constant. En fait le comportement caoutchoutique $E_{\mathrm{c}} / E_{v}$ n'apparaît véritablement que dans des systèmes de masse moléculaire nettement plus élevés que $10^{5}$ daltons. La coupure $n_{\mathrm{c}}$ est donc aussi une fonction de la masse moléculaire.

5. Retour à l'équilibre : relaxation. - Après cessation de la contrainte $\sigma$ (c'est-à-dire $s=0$ ), la chaîne tend à reprendre la configuration d'équilibre.

Soit $\tau$ le temps de relaxation de la chaîne en supposant la structure d'enchevêtrement figée. Soit $\tau_{s}$ le temps de relaxation de la structure. Nous avons vu que $\tau_{s}$ dépend en fait de $i-j=n, \tau_{s}=\tau_{s, n}$. 
a) Expérience de recouvrance partielle : $\tau_{s} \rightarrow \infty$. La longueur de l'échantillon $L$ tend vers $L_{0}$ à partir du moment $t$ où le gradient $s=0$. A l'instant $t+t_{1}$, tel que $\lambda=3 / 2$, on fige la configuration de l'échantillon par trempe rapide.

Dans la figure $8 b$ on a tracé l'intensité diffusée par l'échantillon, à ce stade de la recouvrance. Nous remarquons une valeur $q=\tilde{q}$ de changement de comportement de $S_{1}\left(q^{l l}\right)$ qui ne diffère pas sensiblement de la valeur $q^{*}$ de l'échantillon dont l'allongement est $\lambda=3$. Cette longueur réciproque $\tilde{q}$ ne peut être assimilée à $q^{*} \propto \lambda^{-1} n_{\mathrm{c}}^{-1}$ si nous supposons $n_{\mathrm{c}}$ indépendant de l'allongement. L'identification $\widetilde{q}$ avec $q_{\lambda}^{*} \propto \lambda$ est plus vraisemblable. Au cours de la recouvrance, seules les configurations de la portion $\left(0, q^{*}\right)$ relaxent vers l'équilibre. Les autres ont déjà relaxé. La dépendance en temps de $q^{*}, q_{\lambda}^{*}$ depuis le début de la déformation jusqu'à l'état relaxé ferait apparaître une différence entre l'étape de déformation et l'étape de retour à l'équilibre. On sait, par ailleurs, qu'à l'échelle macroscopique la recouvrance $L \rightarrow L_{0}$ mesurée, est en général plus grande que la recouvrance calculée selon le principe de superposition linéaire.
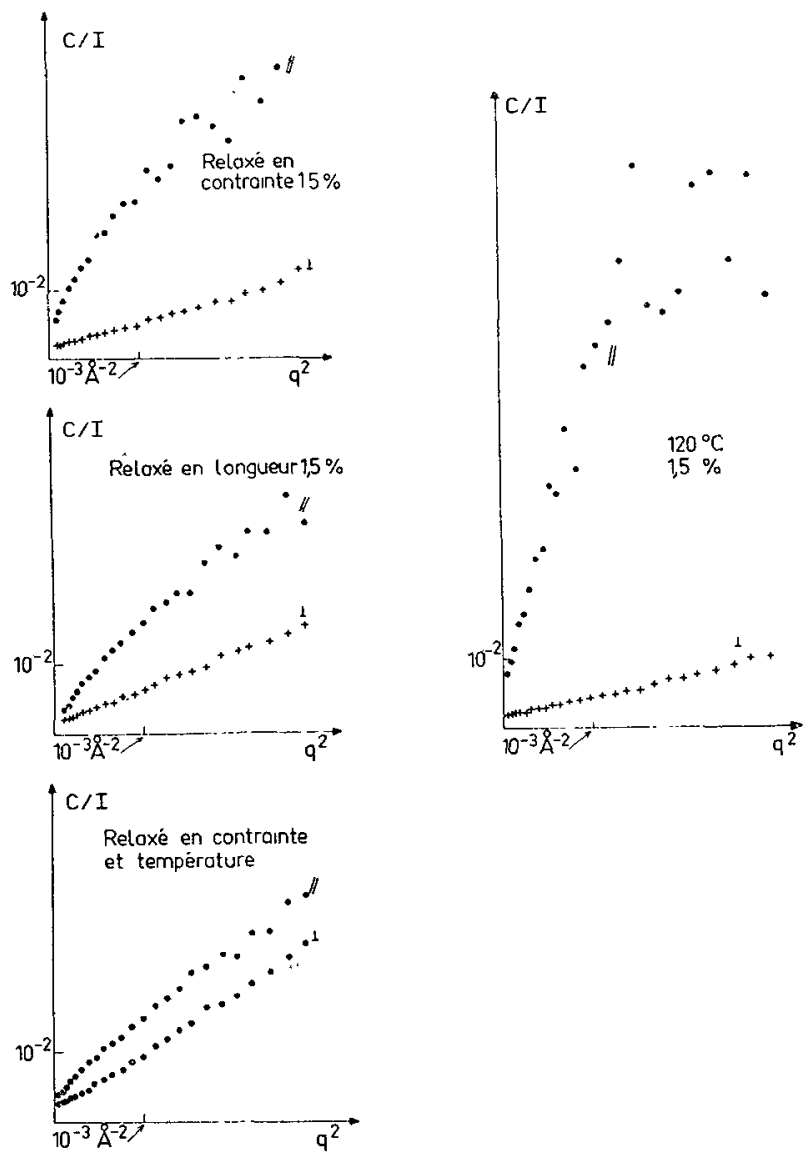

FIG. 8. - Intensités diffusées dans les directions longitudinales et transversales à différents stades de la relaxation. Recouvrance à $\lambda / 2$ (milieu gauche). Relaxation de contrainte à $\sigma / 2$ et $\sigma / 4$ (en haut, en bas à gauche, respectivement). La figure à droite est l'intensité diffusée avant relaxatión. L'allongement de l'échantillon reste fixé à $\lambda=3$. b) Expérience de relaxation à allongement $\lambda$ fixé. La contrainte $\sigma_{0}=E\left(\lambda^{2}-1 / \lambda\right)$ tend alors vers zéro. L'échantillon est figé par trempe rapide à une contrainte prédéterminée $\sigma_{r}<\sigma_{0}$. Les diagrammes de diffusion des neutrons sont portés sur la figure $8 a$ $\left(\sigma_{r}=\sigma_{0} / 2\right)$ et $8 b \quad\left(\sigma_{r}=\sigma_{0} / 4\right)$. La relaxation de contrainte des polymères déformés met en évidence une superposition des effets de temps et de température

$$
S_{1}(q)=S_{1}\left(q, a_{T}^{t} t\right)
$$

où $a_{T}^{t}$ est une fonction de la température $T$ et de la distance réciproque $q^{-1}$.

1) Pour un temps de vie infini du réseau, Daoudi [1] et de Gennes ont montré que pour un temps donné $t$ la quantité significative dans la relaxation de la configuration est $x=n / \sqrt{t / \tau_{1}}$. Soit alors $n_{\text {ccc }}$ : pour $\sqrt{t / \tau_{1}}>n_{\text {ccc }}$, il y a relaxation, pour $\sqrt{t / \tau_{1}}<n_{\text {cce }}$, la configuration n'a pas encore relaxé. La loi d'échelle dans l'espace réciproque est

$$
q^{* *}=\frac{1}{a}\left(\frac{N t}{\tau}\right)^{-1 / 4}
$$

où $\tau$ est le temps caractéristique de relaxation de la chaîne :

$$
\log \tau_{1}=-2,8-\frac{17,44\left(T-T_{\mathrm{B}}\right)}{51,6+T-T_{\mathrm{B}}}+3,4 \log N .
$$

La figure $8 a, c$ montre effectivement une loi $S_{1}(q)$ avec deux comportements distincts séparés par une longueur inverse $q^{* *}$, qui décroît avec le temps $(a)$ et la température $(b)$.

2) Le temps de vie du réseau ne peut être considéré comme infini puisqu'il y a fluage du matériau (déformation plastique). Cependant si le temps de vie du réseau était du même ordre de grandeur que celui de la chaîne, la configuration de la chaîne associée à $\left(0, q^{*}\right)$ relaxerait à la même vitesse que celle qui est associée à la portion $\left(q^{*}, l^{-1}\right)$. Or ceci ne semble pas être le cas. Nous notons donc une grande différence entre $\tau_{1}$ et $\tau_{S}$.

6. Conclusion. - Ces expériences nous apportent :

- Une observation de la configuration de la chaîne déformée, dans l'espace réciproque (A).

- Une observation de la structure du matériau déformé, dans l'espace réciproque (B).

1) Echantillon de type $A$.

a) L'observation de la configuration nous permet de discerner des zones d'espaces réciproque. Les zones sont floues : les longueurs caractéristiques sont des longueurs de contour $n_{\mathrm{c}}$, et $n_{\mathrm{cc}}$. On peut attribuer un intervalle $\left(0, q^{*}\right)$ aux effets d'enchevêtrements : la déformation de la configuration y est essentiellement quadrupolaire. Dans l'intervalle $\left(q^{*}, q_{\lambda}^{*}\right)$ elle est 
dipolaire : la chaîne a tendance à s`aligner dans la direction de l'allongement.

Dans l'intervalle $\left(q_{\lambda}^{*}, l^{-1}\right)$, la configuration est insensible à la déformation.

L'intervalle $\left(0, q^{*}\right)$ est significatif : dans le cas d'une chaîne en solution diluée soumise à un écoulement élongationnel, il n'existe pas.

b) Retour de l'équilibre. Le chemin du retour est différent du chemin de l'aller. L'analyse est rendue plus difficile par la limitation de l'intervalle caractéristique de la déformation ( $q^{*}$ relativement faible). Néanmoins on peut discerner deux intervalles d'espace réciproque $\left(0, q^{* *}\right)\left(q^{* *}, l^{-1}\right)$. La dépendance en temps et en température de $q^{* *}$ nous indique la manière dont la configuration de la chaîne se relaxe.

2) Echantillon de type $B$.

La figure 5 donne un aperçu des possibilités de la méthode d'exploration de la structure de ces matériaux.
Y a-t-il localisation ou non des forces de cohésion pour l'intermédiaire des points d'enchevêtrements ? Des mesures plus précises sont en cours.

Remerciements. - Ces résultats ont été obtenus par le groupe CRM Saclay avec l'aide de Monsieur de Vries (Rhône Poulenc). Nous tenons à remercier tout particulièrement MM. Bonnebat, Daoudi et de Gennes des nombreuses conversations dont nous avons bénéficiées. [J. des Cloizeaux nous fait remarquer que la relation (3.11) n'exprime pas nécessairement une déformation de type dipolaire (cf. la référence Benoit H. et al., Macromolécules 8 (1975) 451). La relation (3.11) correspond en fait au cas particulier où les distances entre points de réticulation ont une distribution Gaussienne [11]. Nous l'en remercions vivement].

\section{Bibliographie}

[1] DaOudi, S., à paraître dans J. Physique (1977).

[2] WARD, I. M., Mechanical properties of Solid Polymers (Wiley Interscience, London, New York, Sydney, Torente).

[3] LodGe, A. S., Elastic Liquids (Academic Press, London, New York).

[4] Ferry, J. D., Viscoelastic Properties of Polymers (John Willey New York, London).

[5] De VRIES, A. J., BonneBat, C. et BeAutemps, J., à paraître dans J. Polym. Sci. C

[6] Duplessix, R., Thèse Univ. Louis Pasteur, Strasbourg (1975).
[7] De Gennes, P. G., communication non publiée.

[8] Cotron, J. P. et al., à paraître.

[9] De GennNes, P. G., J. Physique 31 (1970) 235.

[10] KuHN, V. W. et Grün, F., Kolloid-Z. 248 (1942).

[11] Pearson, D. S., à paraître (1977).

[12] Cleermann, Karam, Williams, Modern Plastics (Breskin Publications Inc. New York) 1953.

[13] Bouk, F., Thèse Univ. Louis Pasteur Strasbourg (1977).

[14] Prcot, C. et al., Macromolécules 10 (1977) 436. 